\title{
The use of MRI to observe fractures in concrete
}

\author{
E. Marfisi*, C. J. Burgoyne*, M. H. G. Amin $†$ and L. D. Hall† \\ University of Cambridge
}

This study extends previous work and demonstrates that magnetic resonance imaging (MRI) of water allows the internal crack pattern of hardened concrete to be observed along with the internal aggregate structure. The ability to reconstruct three-dimensional images is demonstrated and the importance of image registration is discussed. By using aramid fibre-reinforced plastics (AFRP), it is possible to make reinforced concrete specimens that can be measured by MRI. Plain concrete cylinders and others externally confined by AFRP spirals have been tested in compression and scanned after various amounts of load have been applied to show the progress of fracture development. Samples have also been made with straight AFRP rods cast into the concrete which, when pulled out, give bond failures and internal cracking. It is possible not only to observe the fractures, but also to relate them to the internal concrete structure determined by previous scans of the freshly prepared concrete samples.

\section{Introduction}

This is the second of three papers that demonstrate how magnetic resonance imaging (MRI) can be used to study a number of key features of concrete. The first paper $^{1}$ clearly demonstrated that for concrete made with white cement and aggregates such as limestone or quartz, which contain no magnetic elements, MRI can be used to produce three-dimensional (3-D) images which show the location of individual pieces of aggregate. The progress of hardening could be measured by determining the magnetic resonance (MR) response of the water in the concrete. It was also shown that flaws and fractures could be detected by ensuring that they were full of water, but that it was unlikely that the structure and fractures could be measured at the same time in a hardened concrete sample.

This paper reports on the observation of fractures in small cylindrical concrete specimens, with and without external spiral reinforcement. The specimens are first loaded and fractured outside the MRI scanner and, following water saturation, the samples are subsequently scanned in the unloaded state. Tests are also reported for a pull-out specimen in which the images

* Department of Engineering, University of Cambridge, Trumpington St, Cambridge CB2 1PZ, UK.

$\dagger$ Herchel Smith Laboratory for Medicinal Chemistry, University of Cambridge School of Clinical Medicine, Robinson Way, Cambridge CB2 2PZ, UK.

(MCR 41229) Paper received 5 January 2004; last revised 9 September 2004; accepted 8 October 2004 of the resultant internal fractures are combined with images of the structure obtained before loading, to show that the relationship between the fractures and the individual pieces of aggregate can clearly be seen.

The third paper $^{2}$ reports on the testing of a reinforced concrete beam loaded inside the MRI scanner, so that images can be obtained with fractures open to their maximum extent.

\section{Magnetic resonance imaging (MRI)}

MRI discriminates between different materials by measuring their water content and other properties. Each measurement involves the application of a radiofrequency (RF) pulse to a sample located in a strong magnetic field, and determines the water content by measuring the signal generated as the water protons return to their equilibrium state. The spatial discrimination is produced by the use of three sets of magnetic field gradients which induce a variation of the magnetic field over the sample in three dimensions; this alters the frequency of the resulting MR signal. More details can be obtained from the first paper ${ }^{1}$ and the specialist literature. ${ }^{3}$

It is critically important that the homogeneity of the magnetic field is not disturbed by magnetic components in the sample, and it was shown that limestone aggregate and white Portland cement (WPC) are both suitable. It is also necessary that any reinforcement is non-magnetic; normal rebar is clearly unacceptable, and stainless steel, which is normally described as 
non-magnetic, was also found to be unsuitable. For that reason, aramid fibre-reinforced plastic (AFRP) was used in this study as is described in more detail below.

\section{Image properties and acquisition time}

The MR signal is generated from a defined volume within the sample which must be placed inside the RF probe which has an internal diameter of $54 \mathrm{~mm}$. For this study, two-dimensional (2-D) slice images through the sample were made with an in-plane pixel resolution of $156 \times 156 \mu \mathrm{m}$ since this is similar to the minimum size of the cracks to be detected (100-200 $\mu \mathrm{m})$. Since the biggest digital matrix available for image acquisition is $256 \times 256$ pixels, the field of view is $40 \times$ $40 \mathrm{~mm}$.

A series of preliminary experiments was carried out to choose the most suitable slice thickness; $1.25 \mathrm{~mm}$ gives a sufficiently large MR signal for observation of all the concrete components and was used for all measurements reported here. The acquisition of a multiple set of 2-D slices allows a 3-D reconstruction of the sample; those slices can either butt up against each other so that the full volume is covered, or be at discrete intervals so that a series of cross-sections is obtained. A set of $321.25 \mathrm{~mm}$ thick slices fill a field of view that is $40 \mathrm{~mm}$ in each direction.

During image acquisition the MR signals are influenced by stray RF fields which produce noise in the image; the quality of an MR image is defined by its signal-to-noise ratio. To minimise the effect of noise, the MR signal was usually acquired four times and the average value used. The time required for the image acquisition is the product of the number of slices, the size of the digital matrix (in one dimension only), the time taken to make one measurement and the delay time between them (the repetition time $T_{\mathrm{R}}$ ), and the number of image repeats. Table 1 shows the acquisition times required for the images discussed here.

It is desirable to use a specimen as large as possible to limit the scale effects on the behaviour of the concrete. $^{4,5}$ Although it is possible to focus on a particular area of interest in a large sample, it was decided in this study to view the whole cross-section and most of the length of the specimen. For many applications, a 2-D observation is sufficient and it is useful to obtain such an image before undertaking a full 3-D acquisition sequence.

\section{Concrete}

Because of the dimensions of the MRI magnet and the associated RF probe used in this investigation, cylindrical specimens $30 \mathrm{~mm}$ in diameter and $45 \mathrm{~mm}$ in length were used, which is small by comparison with the size of normal structural engineering components. The maximum size of the aggregates was chosen to be in proportion to the dimensions of the specimen; if it is too large, subsequent cracking is strongly influenced by the distribution of the aggregate. If the aggregate is too small, the material behaves like mortar and is more like a continuum whose properties do not reflect the behaviour of a concrete. However, the minimum size of the aggregate must be sufficiently large to allow its clear observation with MRI. The aggregates used here passed a $5 \mathrm{~mm}$ sieve; the concrete proportions used for manufacturing all specimens are shown in Table 2. The aggregate sizes used in these tests are smaller than normal in bulk concrete, but are in proportion to the size of the samples tested and it is believed that the resulting crack patterns are representative. Further studies are underway to determine scale effects, both in the size of the samples and the size of the aggregates used.

A series of compression tests were made to verify the behaviour of the sample. Although it is accepted that the samples may be affected by scale effects, the form of the stress-strain curve and the mode of failure were similar to those of larger specimens. Hence it is believed that the results described here are both an adequate proof-of-concept and of interest in their own right.

Each sample was cast in a thin polythene beaker in which it was left for both compression testing and MRI scanning. This allowed the sample to be kept wet and prevented complete fragmentation when fracture took place. Confined samples were obtained using AFRP spirals anchored to the concrete. Some samples were cast with an AFRP rod placed vertically in the beaker, which was subsequently used for a pull-out test. This rod was wrapped in plastic tape over part of its length to reduce the bonded length so that the rod did not snap when being pulled out.

Table 1. Acquisition time with $256 \times 256$ pixels and four averages

\begin{tabular}{l|c|c}
\hline & 2-D slice & 3-D with 32 slices \\
\hline $\begin{array}{l}\text { Fracture (hardened concrete) } \\
T_{\mathrm{R}}=800 \mathrm{~ms}\end{array}$ & $14 \mathrm{~min}$ & $7 \mathrm{~h} 17 \mathrm{~min}$ \\
Structure (fresh concrete) & $1.7 \mathrm{~min}$ & $55 \mathrm{~min}$ \\
$T_{\mathrm{R}}=100 \mathrm{~ms}$ & & \\
\hline
\end{tabular}

Table 2. Concrete mix

\begin{tabular}{l|c|c}
\hline Material & Size: $\mathrm{mm}$ & Quantity: $\mathrm{kg} / \mathrm{m}^{3}$ \\
\hline Limestone & $5 \cdot 00-2 \cdot 36$ & 769 \\
Sand type B & $1 \cdot 18-0 \cdot 60$ & 673 \\
White cement & - & 625 \\
Deionised water & - & 325 \\
\hline
\end{tabular}




\section{Aramid reinforcement}

The reinforcement used in these tests was made from Kevlar 49 aramid yarns. ${ }^{6}$ Since these yarns do not yield and were not expected to break, their detailed properties are not of great importance; nevertheless, their properties are given in Table 3 . To produce a straight reinforcing bar, ten yarns were coated with epoxy resin, twisted together and held straight in a jig until the epoxy had set. These rods were then tested in a tension testing machine to determine their strength and modulus. Predictably the strength is significantly less than the sum of the strengths of the yarns due to the difficulty in ensuring equal load sharing, but the modulus is closer to that of the yarns, since the loss of stiffness is caused only by misalignment, which is slight. ${ }^{7}$ Such bundle effects are well known ${ }^{8}$ and would be reduced if the rods were made by machine, which would enable better quality control.

The spirals used to provide the lateral confinement of the cylindrical specimens were made in a similar way; six yarns were wrapped around cylindrical formers immediately after twisting them together. Tensile tests were not carried out on these specimens, but it is to be expected that their strength and stiffness would be similar to those of the straight specimens.

The use of FRP reinforcement should not significantly alter the crack patterns obtained; cracking is controlled by the stress field near the crack tip and this

Table 3. Yarn and AFRP properties (based on fibre crosssectional area)

\begin{tabular}{l|c|c|c|c}
\hline & $\begin{array}{c}\text { Tensile } \\
\text { strength: } \\
\mathrm{MPa}\end{array}$ & $\begin{array}{c}\text { Fibre area: } \\
\mathrm{mm}^{2}\end{array}$ & $\begin{array}{c}\text { Elastic } \\
\text { modulus: } \\
\mathrm{GPa}\end{array}$ & $\begin{array}{c}\text { Strength } \\
\text { (as force): } \\
\mathrm{N}\end{array}$ \\
\hline $\begin{array}{l}\text { Kevlar 49 } \\
\text { yarn }\end{array}$ & 2640 & $0.168 \times 10^{-6}$ & 120 & 445 \\
$\begin{array}{l}\text { Ten yarn } \\
\text { AFRP rod }\end{array}$ & 2100 & $1.68 \times 10^{-6}$ & 120 & 3520 \\
\hline
\end{tabular}

should not be affected by the type of reinforcement. There could be some difference in the confined specimens at high loads, when steel spirals would yield and thus apply constant forces while FRP spirals remain elastic and apply increasing force, but this can be taken into account if comparing the crack patterns with a numerical analysis.

\section{Concrete specimens}

The concrete specimens were cast into plastic beakers which were left in place during the testing since they prevented the specimens drying out, and ensured that the fragments did not fall apart after the specimens had been loaded. The beakers were thin and flexible and are not believed to have had significant effect on the behaviour of the samples under load.

Four types of specimens were cast

(a) plain (unreinforced) samples (type A)

(b) samples with spiral reinforcement cast within the concrete (type B)

(c) samples with spiral reinforcement outside the plastic beaker (type C)

(d) samples with a straight AFRP rod to be used for pull-out tests (type D).

Figure 1 shows types A, C and the beaker for type D. Types A, B and C were used successfully for compression tests. However, problems occurred with the sample of type B because of lack of anchorage of the spirals at their ends; as a result the spirals failed to confine the concrete at the ends, which led to premature failure. However by wrapping the spirals around the outside of the plastic beakers as in type $\mathrm{C}$ it was possible to provide more effective end anchorages and, because the concrete was cast directly into the beakers, the spirals were still effective in providing confinement.

The type D pull-out specimens had an AFRP rod cast into the beaker which passed through a hole in the

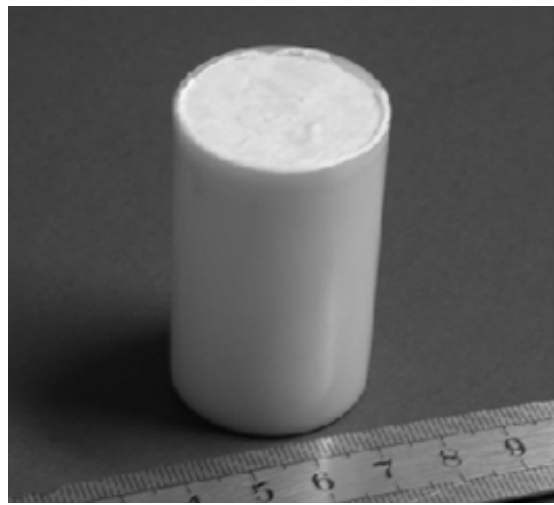

(a)

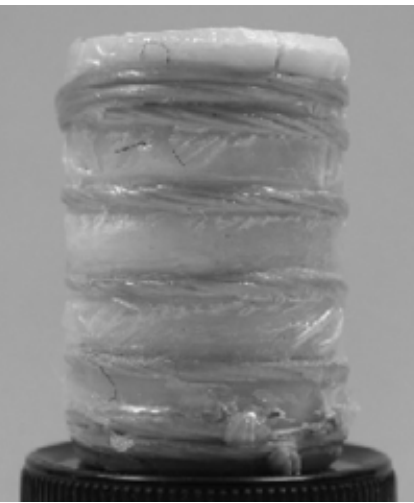

(b)

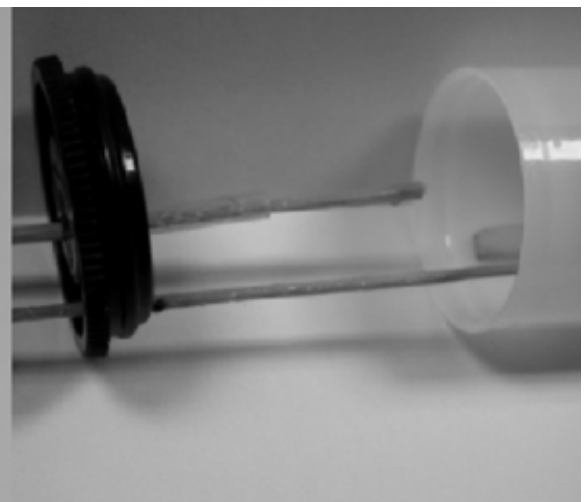

(c)

Fig. 1. Photographs of the three samples: (a) unconfined type A; (b) externally confined type C; and (c) pull-out test type D

Magazine of Concrete Research, 2005, 57, No. 2 
lid of the beaker so that it could be pulled out while still keeping the specimen full of water. In order to reduce the amount of bond, and to enable the rod to be pulled out without breaking it, part of the rod near the lid was debonded by placing a plastic sleeve over it. This is clearly visible on the right hand photograph of Fig. 1 which was taken before the concrete was cast. A second AFRP rod is visible in this photograph but it was not used in the tests. Also just visible in this photograph is a rectangular piece of potato that was placed inside the beaker, as described in the first paper. Being stiff and full of water, the potato provided a spatial registration marker that was visible in all MRI scans of the sample. ${ }^{1}$

\section{Compression tests to generate fractures}

The purpose of these tests was to produce samples which would lead to scans with high image contrast between the solid components and the fractures, and hence to clear-cut observation of the fractures after the concrete has fully hardened. This necessitated filling the fractures with water before each phase of scanning. After testing, the samples were placed under water and in a vacuum before each scanning phase; air bubbles could be seen bubbling through the water and sufficient time for saturation of the sample was estimated experimentally to be $20 \mathrm{~min}$.

Figure 2 shows typical stress-strain curves for con-

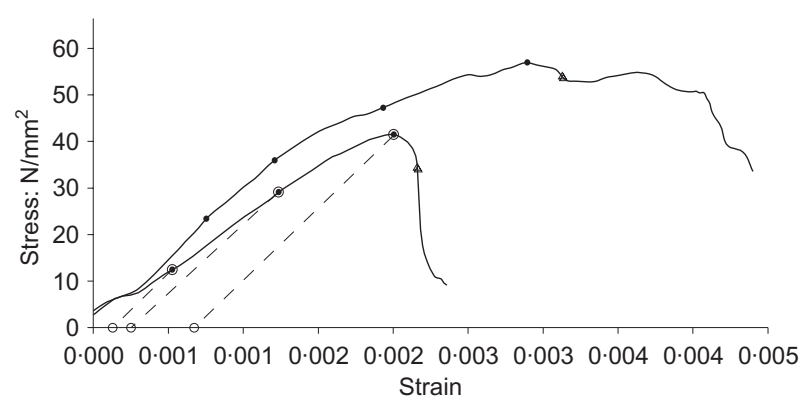

Fig. 2. Typical stress-strain curves for concrete specimens: (a) confined (upper curve); and (b) unconfined (lower curve) fined and unconfined specimens. The unconfined specimens reached a peak load at about $40 \mathrm{MPa}$, with a rapid decrease in load capacity as the strain increased. The confinement made the specimens stronger (about $57 \mathrm{MPa}$ ) and also significantly increased the strain capacity after the peak load.

Data from the literature predict the production of the first micro-cracks at $40 \%$ of the ultimate load. ${ }^{9}$ Thus three different stages of load were chosen to enable observation of different sizes of fractures during a compression test. The specimens were scanned after unloading from the chosen load so the actual stress state while being scanned is represented by the open circles at the base of the dashed unloading lines in Fig. 2.

Figures 3 and 4 show 2-D MRI slice scans taken axially and transversely through a confined specimen. In these, and many of the later figures in this paper, the image intensity has been inverted (so the presence of water is shown as black, instead of white as in the original images), and the range of grey levels has been adjusted in order to aid reproduction for publication. In each case the scan on the left is taken before loading while the next three are from samples after unloading from about $50 \%, 75 \%$ and $100 \%$ of the ultimate load, respectively. No attempt has been made to provide registration between these images, so the four slices in each figure do not necessarily relate to the same slice through the specimen.

It is clear from these images that very little cracking develops until about $75 \%$ of the peak load is reached; nevertheless, the internal cracking structure in the images on the right can clearly be seen.

A comparison between the results from plain and confined concrete highlights the confinement effect and a typical example of these observations is shown in Fig. 5. The differences between them are clear. The unconfined sample has a major fracture propagating at an angle of about $21^{\circ}$ to the loading axis; in contrast, the confined sample shows fractures at about $21^{\circ}$ to the transverse direction which indicates that the passive loading from the confining spirals is also very significant. The transverse scan shows circular and radial fracture surfaces for the unconfined sample, which

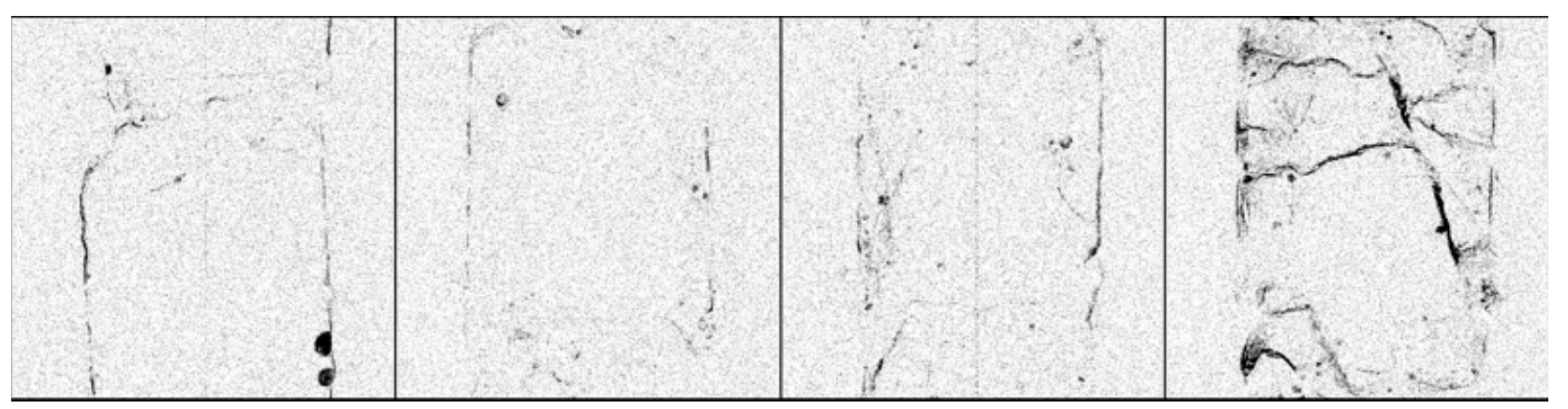

Fig. 3. Progression of fracture for confined samples (slices parallel to the loading direction) 


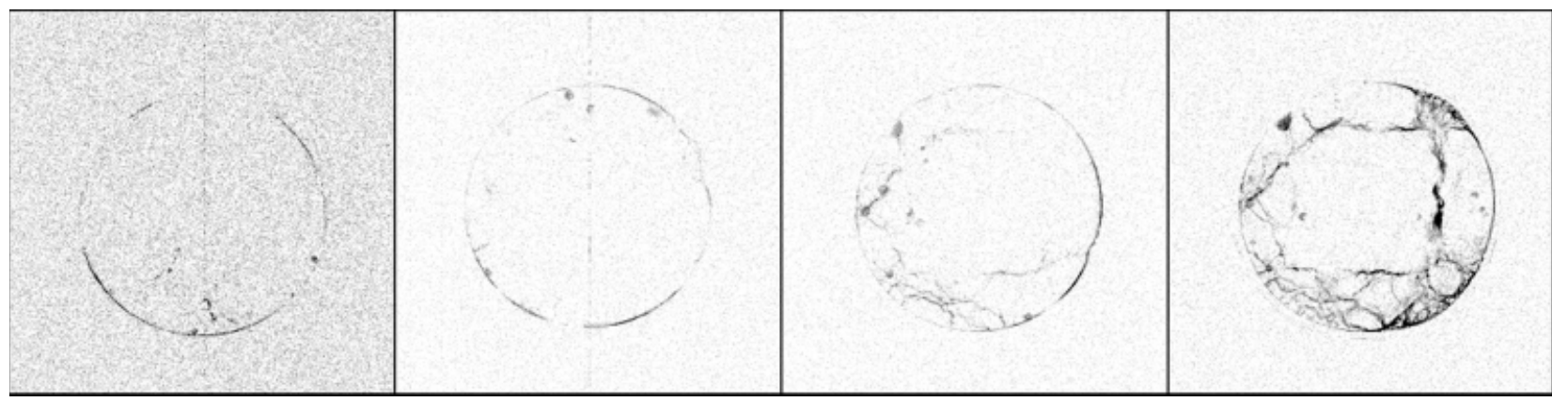

Fig. 4. Progression of fracture for confined samples (slices transverse to the loading direction)

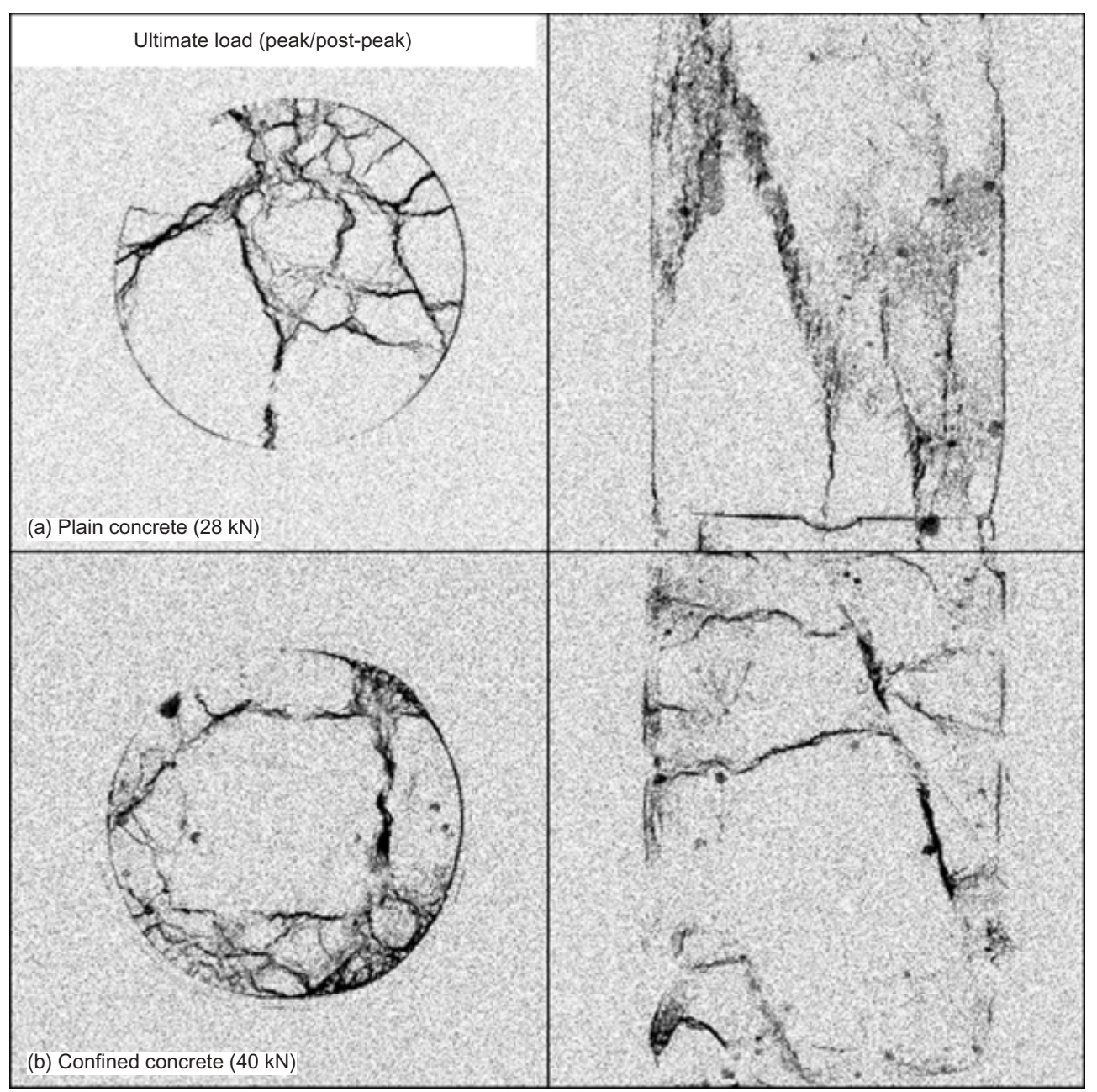

Fig. 5. Images of slices through concrete samples after the ultimate load has been reached: (a) unconfined; and (b) confined

means that the fracture has propagated as a cone from one end, whereas the confined sample shows a square pattern, which is directly related to the location of the reinforcement. Such observations could not be made without visualisation of the internal structure of the fractures and these images are at present unique examples of the power of this MRI methodology.
Although this procedure can be used for a quantitative analysis of the behaviour of various mix designs or forms of confinement, it is more relevant to be able to compare the image of the fracture with that of the concrete structure. Fig. 6 shows the two image forms that can be obtained from the same concrete sample. The one on the left was obtained while the concrete 
Marfisi et al.

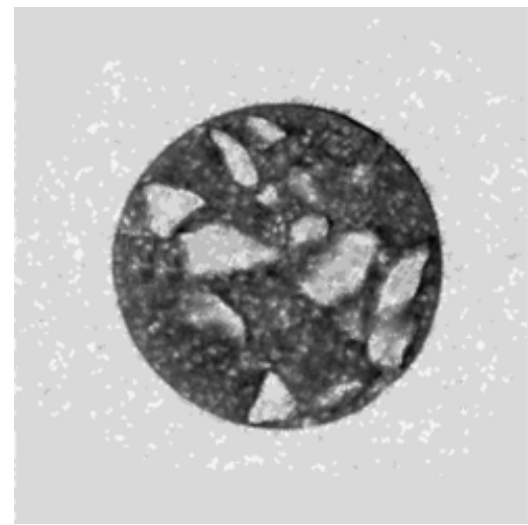

(a)

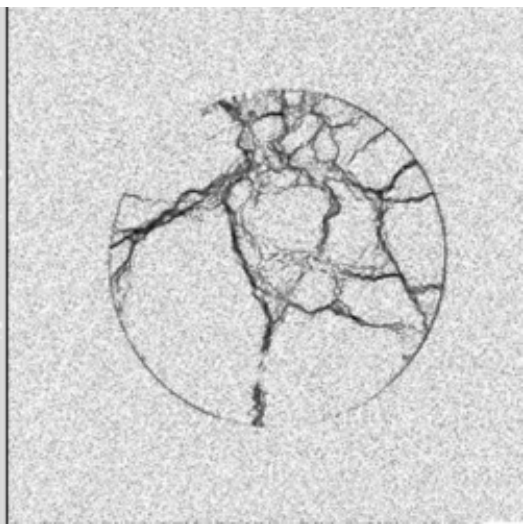

(b)

Fig. 6. Images of: (a) the concrete structure; and (b) the fracture state

was still fresh, and clearly shows the aggregate and sand against the background of the matrix; the image on the right shows the fractures. It is possible to superimpose these two images, using false colour for one of them to distinguish elements that contained water during curing from elements that contain water in a fracture. This superposition has not been carried out here because these images do not refer to the same slice but it is shown for some later images.

\section{Pull-out specimens}

The scanning of the pull-out samples was made under conditions that enabled image-co-registration. Fig. 7 shows what was achieved. These images have not been inverted, nor have the greyscale levels been adjusted. The top row of images show slices through the sample at a level where the rod was bonded to the concrete; the bottom row shows slices where the rod and the concrete were debonded by means of a plastic
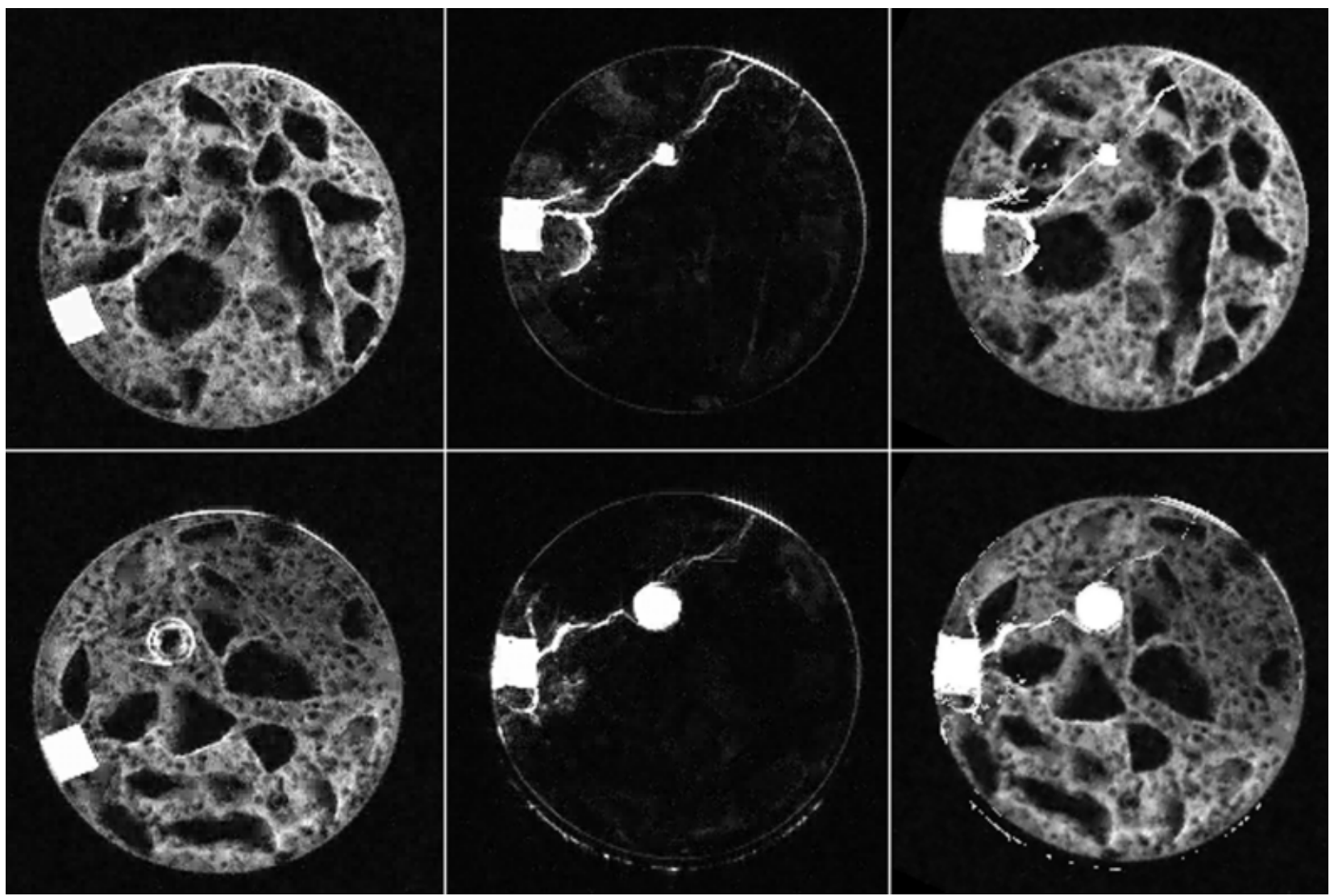

Fig. 7. Pull-out specimen. Top row: slices through sample where rod is bonded to concrete; bottom row: slices through sample where rod is sleeved. In each row, the image on the left was obtained before testing; the central image shows fractures; and the images on the right show the two images co-registered and superimposed 
sleeve. In each row, the image on the left shows the sample as scanned while curing; the AFRP rod shows as a circular black dot since it contains virtually no water, and the potato registration square is also clearly visible on the left-hand side. In the lower image the edges of the plastic sleeve can be seen. The central scans show the sample after it was loaded in a tension testing machine to pull out the AFRP rod. The hole left by the AFRP rod has filled with water, as have both the cracks which join the rod to the surface and the other cracks that occurred internally. The registration square is also clearly visible; the potato itself has shrunk but the space it left has been filled with water. The images on the right shows the two images rotated, registered and superimposed. The relative position of the aggregate and the fractures is clear; some of the fractures skirt round the aggregate pieces, but some of the cracks pass through larger pieces of aggregate. This black and white reproduction is less clear than is possible if colour can be used, since contrasting colours can be assigned to the water in the two images.

It is possible to automate the process of detecting the cracks, as shown in Fig. 8. Fig. 8(a) is from the original sample; a small amount of water present in the matrix shows as dark grey pixels. These can be removed by 'segmenting' the image (selecting only a narrow range of grey levels, and then setting all others to black as in Fig. 8(b)). It is also possible to apply edge detection algorithms to the original image (Fig. 8(c)). Fig. 8(d) shows the effect of applying both of these methods simultaneously and clearly delineates the path of the major fractures. It should be noted that some resolution is lost in this process, and the optimisation parameters that need to be used in different circumstances remain to be studied, but the potential benefits of applying these techniques to large 2-D and 3-D data sets is clear.

\section{Image quality}

The overall quality of an MRI image is directly dependent on the size of the image pixels, their intrinsic signal-to-noise $(\mathrm{S} / \mathrm{N})$ ratio, and the overall scan time. Smaller pixels correspond to higher spatial resolution; however, a decrease in pixel size results in a decrease in the $\mathrm{S} / \mathrm{N}$ ratio which produces a decrease in
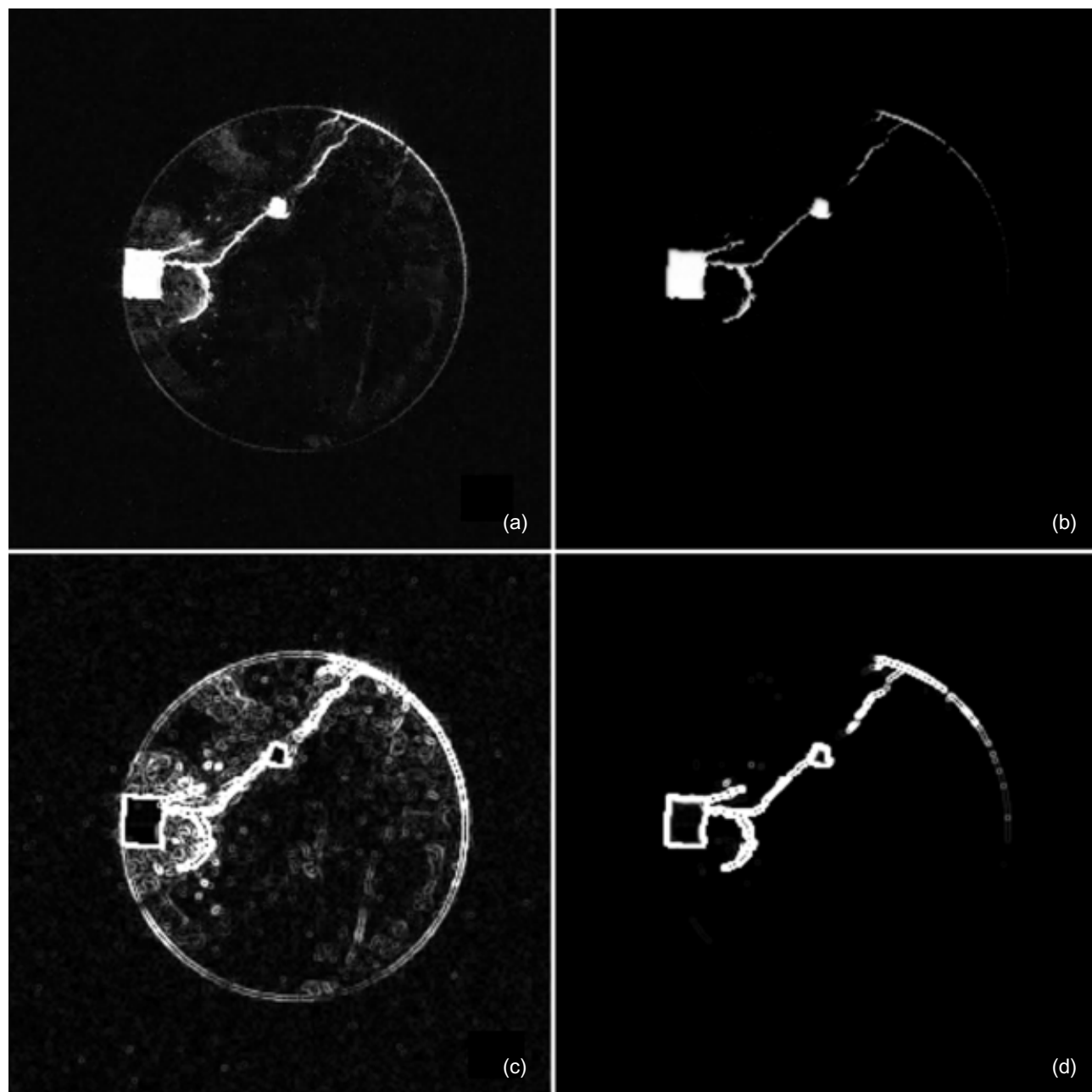

Fig. 8. Automated image analysis; (a) original image; (b) segmented image; (c) edge detection applied to (a); (d) edge detection applied to (b) 
image quality. Increased scan times can enhance image quality in two ways: first, more pixels can be obtained; and second (less obviously), if the same pixels are scanned repeatedly and the signals averaged, this increases the $\mathrm{S} / \mathrm{N}$ ratio of each pixel and hence enhances the overall image quality in proportion to the square root of the number of averages.

Figure 9 shows the original fracture image (on the right), and an expansion of a small portion of that image to show the individual pixels of which it is composed; in addition, four white squares, with sides of 1, 2, 3 and 4 pixels are shown as insets. Each image pixel is $156 \times 156 \mu \mathrm{m}$. Clearly, the size of the fractures can be measured directly from such scans, not only at the surface, which can be measured by a conventional crack microscope, but also internally.

Once the number of single pixels have been selected, the total time required to scan an object is directly related to the repetition time $T_{\mathrm{R}}$ which is, effectively, the time required to take one reading at each voxel. Fig. 10 shows three scans of the same slice taken with three different $T_{\mathrm{R}}$ values. The image on the right is noticeably clearer than the image on the left; however, it took eight times as long to obtain. To provide a more objective measure of this clarity, four regions in the image (A, B, C and D) were chosen to reflect the different fracture types; a region outside the sample $(\mathrm{N})$ was also specified since any signal there is noise. The signal-to-noise ratio was then determined by comparing the strength of the signal in each of the regions $\mathrm{A}, \mathrm{B}, \mathrm{C}$ and $\mathrm{D}$, with that in N. The results of those calculations are shown in Fig. 11. Although the absolute values of the $\mathrm{S} / \mathrm{N}$ ratio are not directly relevant since they depend on the amount of water that is actually present in the particular region, the curves show that there is an improvement in image quality as $T_{\mathrm{R}}$ is increased, but that there is little gain in most cases from increasing $T_{\mathrm{R}}$ much above $800 \mathrm{~ms}$, which was the value chosen for all the measurements in this study (unless otherwise stated). This effect is due to the long lifetime of the magnetisation of the water in the fractures and to the fact that the $\mathrm{S} / \mathrm{N}$ ratio of each pixel is reduced if it is sampled too rapidly.

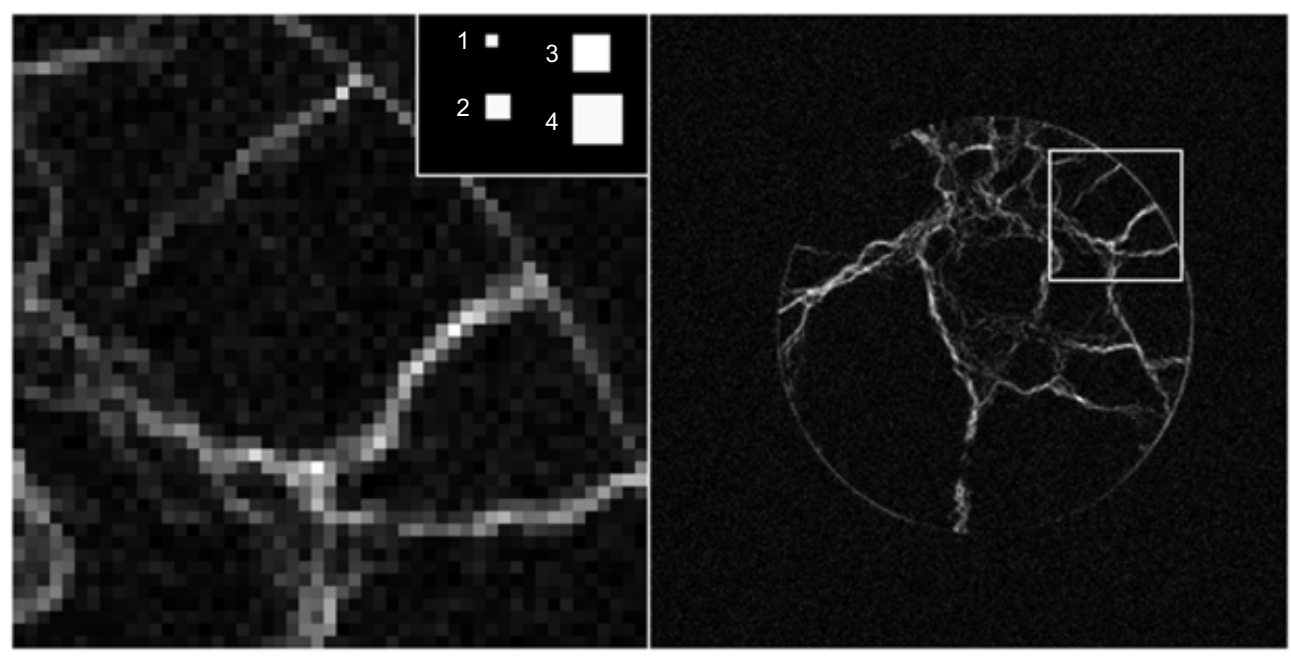

Fig. 9. Detail of fracture image
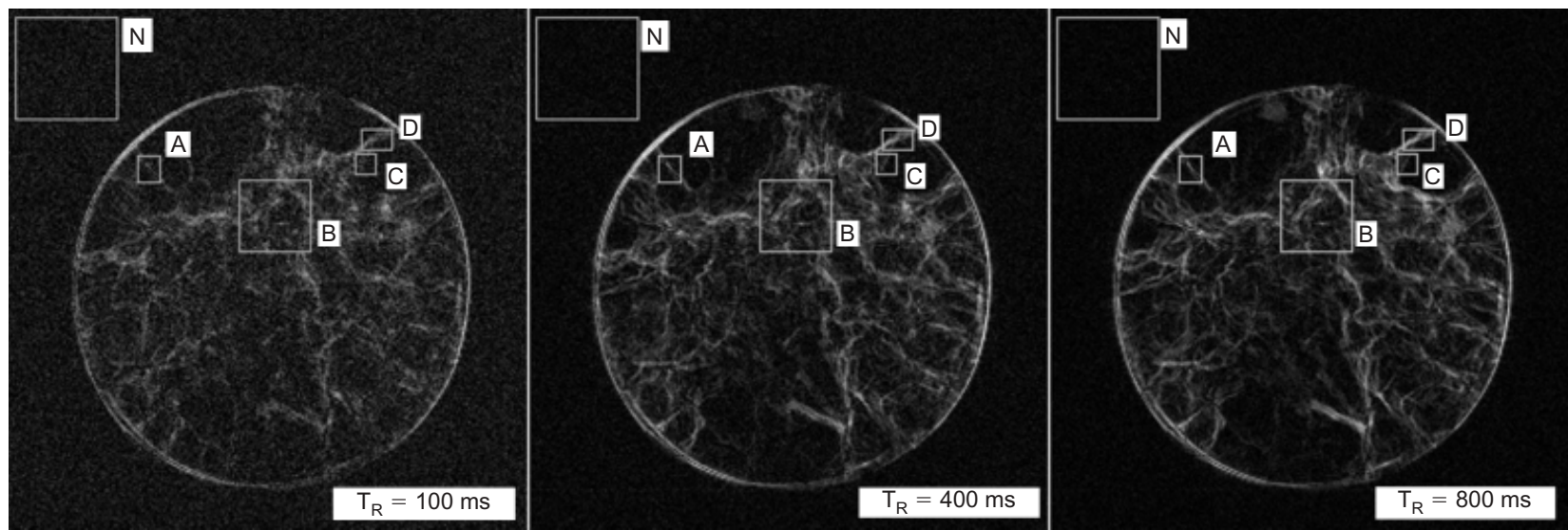

Fig. 10. Images of hardened concrete sample with fractures taken at three different $T_{\mathrm{R}}$ values 


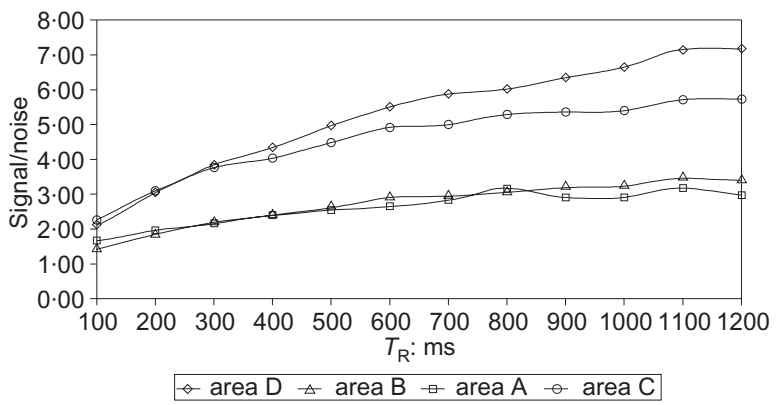

Fig. 11. Signal-to-noise ratios for images with fracture as a function of repetition time $T_{R}$

\section{Three-dimensional reconstruction}

Analysis of 3-D MR images can be achieved either by regarding the data matrix as being composed of a set of 2-D cross-sectional images which can be viewed separately, or by using 3-D reconstruction software to display the entire data matrix. Fig. 12 shows a set of three successive slices taken from the confined compression specimen. Such sets of slices can be combined to make a movie. Alternatively, 3-D image analysis software can be used, for example, to determine continuity between fractures. Three-dimensional reconstruction software allows the data set to be manipulated by the operator so that it is possible to display only those voxels that have specified intensities, and the subsequent image can then be rotated or zoomed to display chosen features, or viewed as a rotating movie so that the full structure can be observed. Images of either the structure or the fractures can be treated separately in this way and it is possible to make the aggregate transparent to show the matrix, and to colour the fractures so that interaction can be seen. Fig. 13 shows images from the same data set, but viewed from different directions. Since printing only allows snapshots of this 3-D image to be viewed, a movie con-

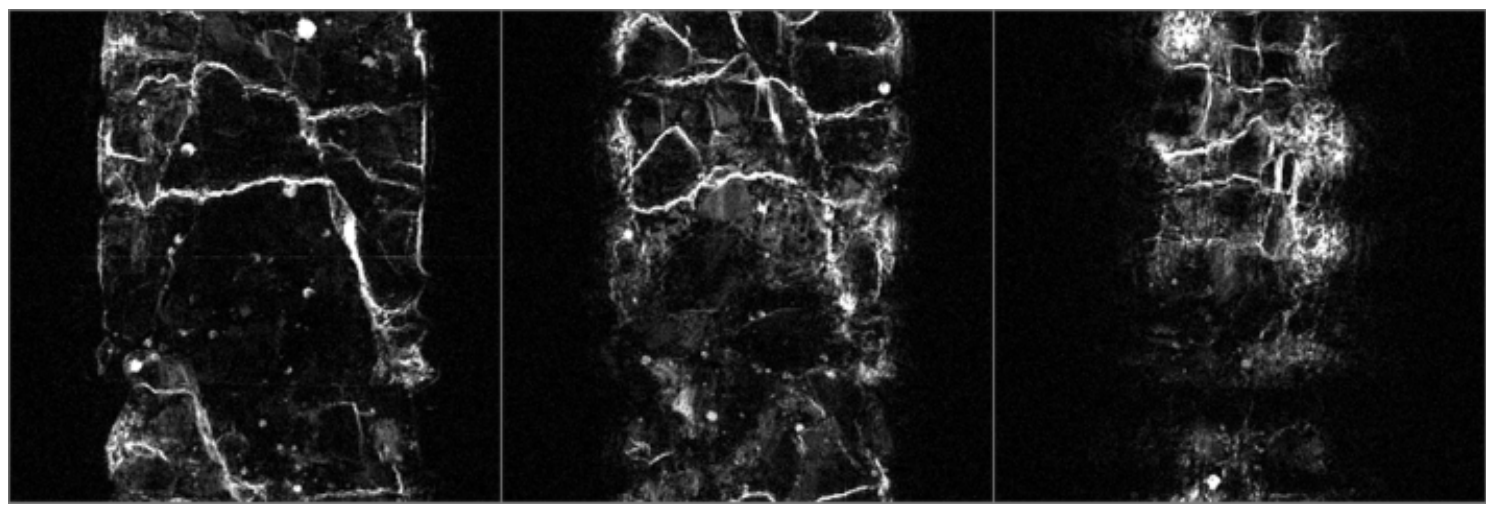

Fig. 12. Longitudinal 2-D slice images of the same sample. The angle with which the spiral follows the cylindrical surface of the sample is evident on the fracture path and is especially clear in the image on the right
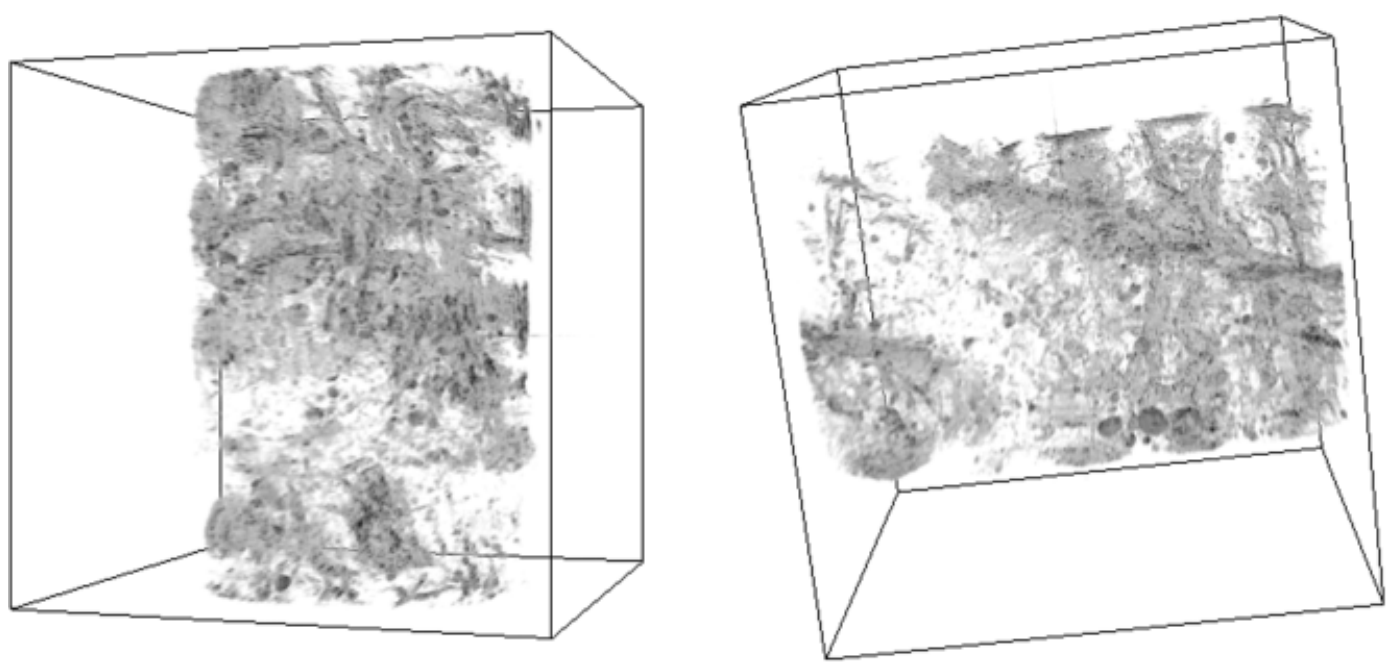

Fig. 13. Three-dimensional reconstruction-fractures within the confined concrete specimen at the ultimate load (two views of the same specimen) 
structed from this data file is available online. ${ }^{10} \mathrm{By}$ moving the image, such factors as the spiral effect of the confinement reinforcement can clearly be seen.

\section{Image analysis software}

A detailed discussion of image analysis software is beyond the scope of this paper since there are so many techniques available for image analysis in two and three dimensions. However, it is appropriate to note that it is possible to count the number and size of individual particles, or to determine automatically the shape, orientation and volumes of complex areas. ${ }^{11}$ Furthermore, it is clear that the quality of the images which can now be obtained is so high that the full potential of MRI for following complex behaviour in concrete will only be realised once the image acquisition described here is matched to image analysis.

\section{Spatial co-registration}

Image co-registration is of crucial importance if the full potential of the MRI technique is to be realised for measurements that require a sample to be scanned, removed for testing, and then returned to the magnet for further scanning. It may be appropriate to repeat this procedure several times. Optimal inter-comparisons of the successive images require cross-correlation of the positions of features between successive images. Although strains are small in concrete samples, gross geometrical changes are possible, so it is also desirable to incorporate MR visible markers in the concrete that will be visible in all images. Small water-filled plastic spheres are possible but their effect on the concrete's properties would need to be ascertained.

Repeated scans may represent the same object from different orientations, in which case the co-registration may only involve a series of translations and rotations of the separate images. However, if the sample geometry or the shape of the elements change it is likely that special software would be needed. Furthermore, this registration software would have to be calibrated to the characteristics of the images and also to the needs of any subsequent analysis.

\section{Beam test}

This preliminary study was intended to demonstrate several proofs-of-concept and it has clearly shown that it is possible to obtain MR images of sufficient quality and resolution for measurements to be taken to support analysis of the mechanics of fracture of both plain and reinforced concrete. To the best of our knowledge such measurements are unique. A companion paper describes the application of these methods to a beam loaded in flexure. ${ }^{2}$ A procedure is described there for loading the sample inside the MRI scanner, filling the cracks with water, and obtaining a succession of images for the entire process.

\section{Conclusions}

Cylindrical concrete specimens, either unreinforced or constrained by spirals of AFRP, have been cast and tested in axial compression and it has been demonstrated that MRI provides a non-destructive method for measuring fractures within a hardened concrete sample. It is feasible for the same sample to be scanned before, during and after a loading sequence, so the progress of fracture generation can be measured. Although this was always intended to be a proof-of-concept study, it nevertheless clearly demonstrates the difference in internal crack structure between plain and reinforced specimens.

Since it is possible to relate images of the fractured samples to those of the concrete structure obtained soon after casting, the relationship between fractures and the aggregate structure within the concrete can be observed. Such comparisons are facilitated if markers are inserted into the concrete samples such that the different images can be accurately related to one another. It is also possible to combine MR images using 3-D image reconstruction software which makes it feasible to study solid models of both the structure and fractures within the concrete.

Thus MRI provides a non-invasive experimental approach to observe the progress of fracture during laboratory testing, and as a result it is now possible to design experiments to measure fracture processes in flexure, shear, bond, confinement or impact. This can be achieved either by using devices designed to load the specimen inside the MRI machine, or by using test procedures that can allow samples to be loaded outside the MRI machine, and then enable the series of independently scanned images to be spatially correlated with one another.

\section{Acknowledgments}

This work was supported by the EU TMR Network 'ConFibreCrete' and by the Herchel Smith Endowments.

\section{References}

1. Marfisi E., Burgoyne C. J., Amin M. H. G. and Hall L. D. The use of MRI to observe the structure of concrete. Magazine of Concrete Research, 2005, 57, No. 2, 103-111.

2. Marfisi E., Burgoyne C. J., Amin M. H. G. and Hall L. D. Observation of flexural cracks in loaded concrete beams using MRI. Magazine of Concrete Research, 2005, 57, (in press).

Magazine of Concrete Research, 2005, 57, No. 2 
3. Callaghan T. Principle of Nuclear Magnetic Resonance Microscopy. Clarendon Press, Oxford, 1991.

4. VAn Mier J. G. M. Fracture Processes of Concrete-Assessment of Material Parameters for Fracture Models. CRC Press, Boca Raton, FL, USA, 1997.

5. Ahmed A. E. Does core size affect strength testing? Concrete International, 1999, 21, No. 8, 35-39.

6. Burgoyne C. J. Aramid fibres for civil engineering applications. In Doran D. K. (ed.) Construction Materials Reference Book, Butterworths, Oxford, 1992.

7. Amaniampong G. and Burgoyne C. J. Statistical variability in the strength and failure strain of aramid and polyester yarns. Journal of Materials Science, 1994, 29, No. 19, 5141-5152.

8. Daniels H. E. The statistical strength of bundles of threads. Proceedings of the Royal Society London, 1945, A183, 45-70.
9. Kotsovos M. D. and Pavlovic M. N. Structural ConcreteFinite Element Analysis for Limit State Design. Thomas Telford, London, 1995.

10. See $<$ http://www-civ.eng.cam.ac.uk/research/mri.html $>$ for further details.

11. Garboczi E. J. Three-dimensional mathematical analysis of particle shape using X-ray tomography and spherical harmonics: Application to aggregates used in concrete. Cement and Concrete Research, 2002, 32, 1621-1638.

Discussion contributions on this paper should reach the editor by 1 September 2005 